\title{
Efficacy of Bio-Rational Insecticides against Sucking Pest of Chilli
}

\author{
P. Prathyusha*, R. K. Dwivedi, Ajay deep singh and Gulab singh \\ Department of Entomology, College of Agriculture, Chandra Shekhar Azad University of \\ Agriculture and Technology, Kanpur-208002, U.P., India \\ *Corresponding author
}

\section{Keywords}

Efficacy, Biorational insecticides, chilli, sucking pest

\section{Article Info}

\section{Accepted:}

20 April 2021

Available Online: 10 May 2021
The present experiment entitled Studies on efficacy of bio-rational insecticides against sucking pest of chilli was carried out in Insectary, Department of Entomology, CSAUA\&T, Kanpur, U.P., during kharif 2018-19 to estimate the efficacy of biorational insecticides against major sucking pest, aphid (A. gossypi), thrips (S. dorsalis, and mite ( $P$. latus) of chilli. Each treatment scheduled to observe the seasonal abundance and two sprays to evaluate the newer and bio-rational insecticides namely, pyproproxyfen 10\% EC @ 0.5ml/1, imidacloprid 17.8 SL @ 0.25ml/1, neem oil 0.03\% EC @ 5ml/l, novuluron+indoxocarb 5.25\%+4.5\% SC @ 0.2ml $/ \mathrm{l}$, verticilium 1.15\%WP@5g/l, cypermethrin 25\% EC @0.5ml/1, emamectin benzoate 5\% SG @ 0.4ml/l, spinosad 45 SC @ 0.5ml/l. Pyproproxyfen 10\% EC @ 0.5ml/1, imidacloprid 17.8 SL@0.25ml/1, novuluron+indoxocarb 5.25\%+4.5\% SC @ 0.2ml/l, and neem oil $0.03 \%$ EC @ 5ml/l were found to be most effective in controlling the aphids, thrips and mite population. Aphid population was effectively controlled by Pyproproxyfen 10\% EC @ 0.5ml/l followed by, imidacloprid 17.8 SL @ $0.25 \mathrm{ml} / 1$, novuluron+indoxocarb 5.25\%+4.5\% SC @ 0.2ml/l. Thrips were significantly reduced by the novuluron+indoxocarb 5.25\%+4.5\% SC @ 0.2 $\mathrm{ml} / 1$ followed by imidacloprid 17.8 SL@0.25ml/1 and neem oil 0.03\% EC @ 5ml/l. The mite population was controlled by imidacloprid 17.8 SL @ 0.25ml/l followed by Pyproproxyfen 10\% EC @ $0.5 \mathrm{ml} / 1$ effectively.

\section{Introduction}

Chilli (Capsicum annuum L.) is a major spice crop cultivated throughout India specially in Andhra Pradesh, Tamilnadu, Karnataka and (Ratnakumari et al., 2001). The native home of chilli is considered to be in Mexico. Capsaicin an alkaloid responsible for pungency in chillies has medicinal properties and it prevents heart attack by dilating the blood vessels (Gill, 1989).

Among the constraints in chilli cultivation, the attack of a multitude of insect pests and mite at different crop stages is of utmost concern. Surveys conducted revealed that, the major pests that attack chilli are aphids (Myzus persicae Sulzer, Aphis gossypi Glover), mite 
(Polyphagotarsonemus latus Banks) and thrips (Scirtothrips dorsalis Hood) (Berke and Sheih, 2000). Patel and Gupta (1992) reported that thrips $S$. dorsalis, cause leaf curling in chilli. Bio-rational insecticides, including botanicals, horticultural oils and products based on entomopathogenic fungi, are alternatives or supplements to chemical insecticides. Over use of pesticides has often led to the development of undesirable problems like destruction of natural enemies, pest resurgence and failure of control strategies leading to heavy damage in chilli.

The presence of pesticide residues in chillies has been of more concern for export of chillies to developed countries. Noval insecticides provides an alternate to the chemical pesticides and to reduce ill effects of conventional pesticides (Joia et al., 2001).

The noval insecticides are more tissuespecific, activated in unique ways inside the target cells of insects resulting in reduced threat to other organism. Selective toxicity to insects and safety to natural enemies have made the new class of insecticides more user and eco-friendly (Gundannavar et al., 2006).

\section{Materials and Methods}

An experiment is conducted in Insectary, Department of Entomology, CSAUA\&T, Kanpur in Kharif season 2018-19. The chilli seedlings of variety Azad-1 was obtained from Vegetable Research farm, kalyanpur, Chandra Shekhar Azad University of Agriculture and Technology. About 550 seedlings are obtained and transplanted in the experimental plot $(2 \mathrm{~m}$ $\mathrm{x} 1.5 \mathrm{~m})$.

The efficacy of the bio-rational pesticides against the sucking pest complex and chilli mite is estimated by recording the pretreatment readings before spraying according to abundance, pest damage and post treatment readings are taken on $7^{\text {th }}$ and $15^{\text {th }}$ days after spraying.

\section{Spraying of Bio-rational insecticides}

The first spraying is done 53 days after transplanting on 13-10-2018 followed by the second spraying is done at 15 days interval on 28-10-2018.

The insecticides which are required in fewer quantities are calculated and measured through micropipette and electronic balance. Then insecticide is diluted with the water in 1.5 lit capacity of hand spray. Spraying is done with the hand pneumatic sprayer.

\section{Preparation of spray fluid}

The insecticidal solution or spray fluid is calculated by using the following formula

Amount of spray fluid required

Conc. Required (\%)

$\mathrm{x}$ Vol. required(1)

Conc. Of toxicant in

insecticidal Formulation

$*($ conc.$=$ concentration, vol. $=$ volume $)$

\section{Results and Discussion}

The experiment was carried out in Kharif during 2018-19 to estimate the efficacy of biorational insecticides against major sucking pest, aphid (A. gossypi), thrips (S. dorsalis) and mite $(P$. latus) of chilli and population dynamics of these pests are noticed in accordance to weather parameters.

Two sprayings are done on appearance of pest. Data is taken during $7^{\text {th }}$ and $15^{\text {th }}$ days after spraying. The percent reduction of insect pest and the generated data was processed statistically. 
Efficacy of bio-rational insecticides on aphid (A. gossypi)

Before spraying, the recorded data indicated that there was no significant difference between populations of different experimental plots showing homogenous pest distribution. The aphid population before spray ranged from 35.04 to 39.43 aphid/ 3 leaves.

\section{Effect of first spray}

After first spray, the data was recorded on 7 days after spraying, according to the data, Pyroproxyfen @ $0.5 \mathrm{ml} / 1$ insecticide was proven most effective in reducing the aphid mean population from 37.57 aphids $/ 3$ leafs to 12.96 over untreated with the reduction percentage of 77.91 followed by mixture of novuluron + indoxocarb @ 0.2mi $/ 1$ was found effective in reducing the mean aphid population from 35.88-16.24 over untreated. The efficacy of all the insecticides decreased after 15 days of applications, though they were significantly superior over untreated control.

\section{Effect of second spray}

After second spray, pyroproxyfen @ 0.5mg/l has proven to be most effective with the lowest mean aphid population of 8.70 and reduction percentage of 87.69 spraying after 7 days. Higher reduction of aphid population was recorded in the pyroproxyfen@5ml/1 treated plot, followed by novuluron + indoxocarb @ 0.2ml/1, imidacloprid @ $0.25 \mathrm{ml} / \mathrm{l}$. Verticilium @ 0.4g/l, neem oil @ $5 \mathrm{ml} / 1$, emamectin benzoate @ $0.4 \mathrm{ml} / 1$ are moderately effective. While, spinosad @ $0.5 \mathrm{ml} / 1$, and Cypermethrin @ 0.5 ml $/ 1$, are less effective in controlling the aphid population.

The obtained results are in agreement with the findings of, Hemant Swami, et al., (2018) reported Pyriproxyfen 10\% EC @ $1250 \mathrm{ml} / \mathrm{ha}$ followed by Imidacloprid $17.8 \%$ SL $50 \mathrm{~g}$ a.i. @ $250 \mathrm{ml} / \mathrm{ha}$ found the most effective to reduce the aphids Aphis gosypii population in chilli crop during both years, Kharif 2016 and 2017. Kens, Stewart (2013) found that Pyroproxyfen affects the population structure and may have potential in managing aphid outbreaks. Patel (2013) found that pyroproxyfen + fenpropethrin $500 \mathrm{ml} / \mathrm{ha}$ was most effective to reducing the aphid population. Whereas Patil et al., (2002) and Kumar et al.,(2015), reported highest yield of chilli with the imidacloprid treatment.

Table.1 Treatment details

\begin{tabular}{|c|c|c|c|c|}
\hline \multirow{2}{*}{ S. No. } & \multicolumn{2}{|c|}{ Treatments } & \multicolumn{2}{c|}{ Dosage } \\
\cline { 2 - 5 } & Common Name & Trade name & Formulation (ml/g/ha) & Dose \\
\hline $\mathrm{T}_{1}$ & Verticilium & Vertimec & $1.15 \% \mathrm{WP}$ & $5 \mathrm{~g} / \mathrm{l}$ \\
\hline $\mathrm{T}_{2}$ & Cypermethrin & Cymbush & $25 \%$ & $0.5 / 1$ \\
\hline $\mathrm{T}_{3}$ & Emamectin & Spolite & $5 \% \mathrm{SG}$ & $0.4 \mathrm{ml} / 1$ \\
\cline { 2 - 5 } & Benzoate & & & \\
\hline $\mathrm{T}_{4}$ & Pyriproxyfen & Lano & $10 \% \mathrm{EC}$ & $0.5 \mathrm{mg} / 1$ \\
\hline $\mathrm{T}_{5}$ & Neem oil & Nimbicidine & $0.03 \% \mathrm{EC}$ & $5 \mathrm{ml} / 1$ \\
\hline $\mathrm{T}_{6}$ & Novoluron+ & Plethora & $5.25 \%+$ & $0.2 \mathrm{ml} / 1$ \\
\cline { 5 - 6 } & Indoxocarb & & $4.5 \% \mathrm{SC}$ & \\
\hline $\mathrm{T}_{7}$ & Imidacloprid & Confidor & $17.8 \mathrm{SL}$ & $0.25 \mathrm{ml} / 1$ \\
\hline $\mathrm{T}_{8}$ & Spinosad & Tracer & $45 \mathrm{SC}$ & $0.5 \mathrm{ml} / 1$ \\
\hline $\mathrm{T}_{9}$ & Control & --- & --- & -- \\
\hline
\end{tabular}


Table.2 Efficacy of bio-rational insecticides on aphid (Aphis gossypi) in chilli crop

\begin{tabular}{|c|c|c|c|c|c|c|c|c|c|c|c|}
\hline \multicolumn{12}{|c|}{ No of aphids/3 leaves } \\
\hline $\begin{array}{c}\text { S.N } \\
\cdot\end{array}$ & Treatment & Dose & DBS & 7 DAS & $\begin{array}{l}\% \text { Reduction } \\
\text { over control }\end{array}$ & 15 DAS & $\begin{array}{l}\text { \% Reduction } \\
\text { over control }\end{array}$ & 7 DAS & $\begin{array}{l}\% \text { Reduction } \\
\text { over control }\end{array}$ & $\begin{array}{c}15 \\
\text { DAS }\end{array}$ & $\begin{array}{l}\% \text { Reduction } \\
\text { over control }\end{array}$ \\
\hline \multirow[t]{2}{*}{1} & \multirow{2}{*}{ Verticilium } & \multirow{2}{*}{$5 \mathrm{~g} / 1$} & 39.43 & 21.99 & \multirow[t]{2}{*}{62.51} & 25.7 & \multirow[t]{2}{*}{61.77} & 17.72 & \multirow[t]{2}{*}{74.94} & 15.68 & \multirow[t]{2}{*}{79.79} \\
\hline & & & $(6.28)$ & $(4.69)$ & & $(5.07)$ & & $(4.21)$ & & $(3.96)$ & \\
\hline \multirow[t]{2}{*}{2} & \multirow[t]{2}{*}{ cypermethrin } & \multirow[t]{2}{*}{$200 \mathrm{~g} / 1$} & 35.04 & 32.71 & \multirow[t]{2}{*}{44.24} & 36.48 & \multirow[t]{2}{*}{45.74} & 28.4 & \multirow[t]{2}{*}{59.84} & 27.66 & \multirow[t]{2}{*}{64.36} \\
\hline & & & $(5.92)$ & $(5.72)$ & & $(6.04)$ & & $(5.33)$ & & $(5.26)$ & \\
\hline \multirow[t]{2}{*}{3} & \multirow{2}{*}{$\begin{array}{c}\text { Emamectin } \\
\text { benzoate }\end{array}$} & \multirow[t]{2}{*}{$0.4 \mathrm{ml} / 1$} & 38.81 & 27.24 & \multirow[t]{2}{*}{53.57} & 30.91 & \multirow[t]{2}{*}{54.03} & 22.94 & \multirow[t]{2}{*}{67.56} & 20.7 & \multirow[t]{2}{*}{73.32} \\
\hline & & & $(6.23)$ & $(5.22)$ & & $(5.56)$ & & $(4.79)$ & & $(4.55)$ & \\
\hline \multirow[t]{2}{*}{4} & \multirow[t]{2}{*}{ Pyroproxyfen } & \multirow[t]{2}{*}{$0.5 \mathrm{mg} / \mathrm{l}$} & 37.57 & 12.96 & \multirow[t]{2}{*}{77.91} & 16.72 & \multirow[t]{2}{*}{75.13} & 8.7 & \multirow[t]{2}{*}{73.85} & 5.66 & \multirow[t]{2}{*}{92.7} \\
\hline & & & $(6.13)$ & $(3.6)$ & & $(4.09)$ & & $(2.95)$ & & (2.38) & \\
\hline \multirow[t]{2}{*}{5} & \multirow[t]{2}{*}{ Neem oil } & \multirow[t]{2}{*}{$5 \mathrm{ml} / 1$} & 38.06 & 22.65 & 61.39 & 26.41 & 60.72 & 18.49 & 73.85 & 16.72 & 78.45 \\
\hline & & & $(6.17)$ & $(4.76)$ & & $(5.14)$ & & $(4.3)$ & & $(4.09)$ & \\
\hline 6 & Novuluron+ & $0.2 \mathrm{ml} / 1$ & 35.88 & 16.24 & 72.31 & 19.98 & 70.28 & 11.97 & 83.07 & 8.7 & 88.79 \\
\hline & Indoxocarb & & $(5.99)$ & $(4.03)$ & & $(4.47)$ & & $(3.46)$ & & $(2.95)$ & \\
\hline 7 & Imdacloprid & $0.25 \mathrm{ml} / 1$ & 35.64 & 18.74 & 68.05 & 22.46 & 66.59 & 14.44 & 79.58 & 11.22 & 85.54 \\
\hline & & & $(5.97)$ & (4.33) & & $(4.74)$ & & $(3.8)$ & & $(3.35)$ & \\
\hline 8 & Spinosad & $0.5 \mathrm{ml} / 1$ & 37.08 & 30.69 & 47.69 & 34.45 & 48.76 & 26.41 & 62.65 & 24.2 & 68.81 \\
\hline & & & $(6.09)$ & $(5.54)$ & & $(5.87)$ & & $(5.14)$ & & $(4.92)$ & \\
\hline 9 & Untreated & -- & 37.33 & 58.67 & 0 & 67.24 & 0 & 70.72 & 0 & 77.61 & 0 \\
\hline & & & $(6.11)$ & (7.66) & & $(8.2)$ & & $(8.41)$ & & $(8.81)$ & \\
\hline & $\mathrm{CD}(\mathrm{P}=0.05)$ & & NS & 0.324 & & 0.3146 & & 0.2765 & & 0.2991 & \\
\hline & $\mathrm{SE}(\mathrm{m}) \pm$ & & 0.114 & 0.1483 & & 0.1118 & & 0.1612 & & 0.1956 & \\
\hline
\end{tabular}

DBS-Days before spraying, DA-Days after spraying, NS-Non significant *figures in parentheses $\sqrt{x+0.5} \sqrt{x+0.5}$ transformed values 
Table.3 Efficacy of bio-rational insecticides on thrips (Scirtothirps dorsalis Hood) in chilli crop

\begin{tabular}{|c|c|c|c|c|c|c|c|c|c|c|c|}
\hline \multicolumn{12}{|c|}{ No.Of thrips / 3 leaves } \\
\hline \multicolumn{8}{|c|}{ First Spray } & \multicolumn{4}{|c|}{ Second Spray } \\
\hline S.N. & Treatment & Dose & DBS & 7 DAS & $\begin{array}{l}\% \text { Reduction } \\
\text { over control }\end{array}$ & 15 DAS & $\begin{array}{l}\text { \% Reduction } \\
\text { over control }\end{array}$ & 7 DAS & $\begin{array}{l}\text { \% Reduction } \\
\text { over control }\end{array}$ & 15 DAS & $\begin{array}{l}\text { \% Reduction } \\
\text { over control }\end{array}$ \\
\hline \multirow[t]{2}{*}{1} & \multirow[t]{2}{*}{ Verticilium } & \multirow[t]{2}{*}{$5 g / 1$} & 12.74 & 9.00 & \multirow[t]{2}{*}{47.73} & 10.69 & \multirow[t]{2}{*}{45.76} & 7.72 & \multirow[t]{2}{*}{65.91} & 6.25 & \multirow[t]{2}{*}{75.68} \\
\hline & & & $(3.57)$ & $(3.00)$ & & $(3.27)$ & & $(2.78)$ & & $(2.50)$ & \\
\hline \multirow[t]{2}{*}{2} & \multirow[t]{2}{*}{ cypermethrin } & \multirow[t]{2}{*}{$200 \mathrm{~g} / \mathrm{l}$} & 14.21 & 12.25 & \multirow[t]{2}{*}{28.86} & 10.49 & \multirow[t]{2}{*}{46.77} & 7.45 & \multirow[t]{2}{*}{67.10} & 5.95 & \multirow[t]{2}{*}{76.84} \\
\hline & & & (3.77) & $(3.50)$ & & $(3.24)$ & & $(2.73)$ & & $(2.44)$ & \\
\hline \multirow[t]{2}{*}{3} & \multirow{2}{*}{$\begin{array}{l}\text { Emamectin } \\
\text { benzoate }\end{array}$} & \multirow[t]{2}{*}{$0.4 \mathrm{ml} / 1$} & 13.69 & 10.69 & \multirow[t]{2}{*}{37.92} & 9.00 & \multirow[t]{2}{*}{54.33} & 5.95 & \multirow[t]{2}{*}{73.73} & 4.49 & \multirow[t]{2}{*}{82.52} \\
\hline & & & $(3.70)$ & $(3.27)$ & & $(3.00)$ & & $(2.44)$ & & $(2.12)$ & \\
\hline \multirow[t]{2}{*}{4} & \multirow[t]{2}{*}{ Pyroproxyfen } & \multirow[t]{2}{*}{$0.5 \mathrm{mg} / \mathrm{l}$} & 15.44 & 7.72 & \multirow[t]{2}{*}{55.16} & 5.95 & \multirow[t]{2}{*}{69.81} & 2.99 & \multirow[t]{2}{*}{86.79} & 1.00 & \multirow[t]{2}{*}{96.10} \\
\hline & & & (3.93) & $(2.78)$ & & $(2.44)$ & & $(1.73)$ & & $(1.00)$ & \\
\hline \multirow[t]{2}{*}{5} & Neem oil & $5 \mathrm{ml} / 1$ & 11.97 & 10.24 & 40.53 & 8.46 & 57.07 & 5.47 & 75.84 & 4.00 & 84.43 \\
\hline & & & $(3.46)$ & $(3.20)$ & & $(2.91)$ & & $(2.34)$ & & $(2.00)$ & \\
\hline 6 & Novuluron+ & $0.2 \mathrm{ml} / 1$ & 14.74 & 7.23 & 58.01 & 5.47 & 72.24 & 2.49 & 89.00 & 1.00 & 96.10 \\
\hline & Indoxocarb & & (3.84) & $(2.69)$ & & $(2.34)$ & & $(1.58)$ & & $(1.00)$ & \\
\hline 7 & Imdacloprid & $0.25 \mathrm{ml} /$ & 12.46 & 8.70 & 49.47 & 6.96 & 64.68 & 4.00 & 82.33 & 2.49 & 90.31 \\
\hline & & 1 & (3.53) & $(2.95)$ & & $(2.64)$ & & $(2.00)$ & & $(1.58)$ & \\
\hline 8 & Spinosad & $0.5 \mathrm{ml} / 1$ & 11.97 & 11.22 & 34.84 & 9.00 & 54.33 & 6.45 & 71.52 & 4.97 & 81.36 \\
\hline & & & $(3.46)$ & $(3.35)$ & & $(3.00)$ & & $(2.54)$ & & $(2.23)$ & \\
\hline 9 & Untreated & -- & 13.46 & 17.22 & 0.00 & 19.71 & 0.00 & 22.65 & 0.00 & 25.70 & 0.00 \\
\hline & & & (3.67) & $(4.15)$ & & $(4.15)$ & & $(4.76)$ & & $(5.07)$ & \\
\hline & $\mathrm{CD}(\mathrm{P}=0.05)$ & & NS & 0.31 & & 0.36 & & 0.21 & & 0.31 & \\
\hline & $\mathrm{SE}(\mathrm{m}) \pm$ & & 0.15 & 0.10 & & 0.12 & & 0.18 & & 0.16 & \\
\hline
\end{tabular}

DBS-Days before spraying, DAS-Days after spraying, NS-Non significant $*$ figures in parentheses $\sqrt{x+0.5} \sqrt{x+0.5}$ transformed values 
Table.4 Efficacy of bio-rational insecticides on mite (Polyphagotarsonemus latus) in chilli crop

\begin{tabular}{|c|c|c|c|c|c|c|c|c|c|c|c|}
\hline \multicolumn{12}{|c|}{ No. of mites / leaves } \\
\hline \multicolumn{8}{|c|}{ First Spray } & \multicolumn{4}{|c|}{ Second Spray } \\
\hline S.N & Treatment & Dose & DBS & 7 DAS & $\begin{array}{l}\text { \% Reduction } \\
\text { over control }\end{array}$ & 15 DAS & $\begin{array}{l}\text { \% Reduction } \\
\text { over control }\end{array}$ & $\begin{array}{c}7 \\
\text { DAS }\end{array}$ & $\begin{array}{l}\% \text { Reduction } \\
\text { over control }\end{array}$ & $\begin{array}{c}15 \\
\text { DAS }\end{array}$ & $\begin{array}{l}\% \text { Reduction } \\
\text { over control }\end{array}$ \\
\hline \multirow[t]{2}{*}{1} & \multirow[t]{2}{*}{ Verticilium } & \multirow[t]{2}{*}{$5 \mathrm{~g} / 1$} & 17.22 & 14.74 & \multirow[t]{2}{*}{54.93} & 16.24 & \multirow[t]{2}{*}{54.88} & 13.98 & \multirow[t]{2}{*}{64.99} & 10.95 & \multirow[t]{2}{*}{70.94} \\
\hline & & & $(4.51)$ & $(3.84)$ & & $(4.03)$ & & (3.74) & & $(3.31)$ & \\
\hline \multirow[t]{2}{*}{2} & \multirow[t]{2}{*}{ Cypermethrin } & \multirow[t]{2}{*}{$200 \mathrm{~g} / \mathrm{l}$} & 20.79 & 17.72 & \multirow[t]{2}{*}{45.82} & 19.18 & \multirow[t]{2}{*}{46.72} & 16.97 & \multirow[t]{2}{*}{57.51} & 13.98 & \multirow[t]{2}{*}{62.90} \\
\hline & & & $(4.56)$ & $(4.21)$ & & $(4.38)$ & & $(4.12)$ & & $(3.74)$ & \\
\hline \multirow[t]{2}{*}{3} & \multirow{2}{*}{$\begin{array}{l}\text { Emamectin } \\
\text { benzoate }\end{array}$} & \multirow[t]{2}{*}{$0.4 \mathrm{ml} / 1$} & 22.46 & 15.68 & \multirow[t]{2}{*}{52.06} & 17.22 & \multirow[t]{2}{*}{52.16} & 14.97 & \multirow[t]{2}{*}{62.51} & 11.97 & \multirow[t]{2}{*}{68.24} \\
\hline & & & $(4.74)$ & $(3.96)$ & & $(4.15)$ & & $(3.87)$ & & $(3.46)$ & \\
\hline \multirow[t]{2}{*}{4} & \multirow[t]{2}{*}{ Pyroproxyfen } & \multirow[t]{2}{*}{$0.5 \mathrm{mg} / 1$} & 27.14 & 11.22 & \multirow[t]{2}{*}{65.69} & 12.74 & \multirow[t]{2}{*}{64.61} & 9.98 & \multirow[t]{2}{*}{49.98} & 6.96 & \multirow[t]{2}{*}{81.53} \\
\hline & & & $(5.21)$ & $(3.35)$ & & $(3.57)$ & & $(3.16)$ & & $(2.64)$ & \\
\hline \multirow[t]{2}{*}{5} & Neem oil & $5 \mathrm{ml} / 1$ & 20.79 & 12.74 & 61.05 & 14.21 & 60.52 & 11.97 & 70.03 & 9.00 & 76.12 \\
\hline & & & $(4.57)$ & $(3.57)$ & & $(3.77)$ & & $(3.46)$ & & $(3.00)$ & \\
\hline 6 & Novuluron+ & $0.2 \mathrm{ml} / 1$ & 18.66 & 11.97 & 63.40 & 13.46 & 62.61 & 11.22 & 71.90 & 8.23 & 78.16 \\
\hline & Indoxocarb & & $(4.32)$ & $(3.46)$ & & $(3.67)$ & & $(3.35)$ & & $(2.87)$ & \\
\hline 7 & Imdacloprid & $0.25 \mathrm{ml} / 1$ & 22.84 & 8.23 & 74.83 & 9.73 & 72.97 & 6.96 & 82.57 & 4.70 & 87.52 \\
\hline & & & $(4.78)$ & $(2.87)$ & & $(3.12)$ & & $(2.64)$ & & $(2.17)$ & \\
\hline 8 & Spinosad & $0.5 \mathrm{ml} / 1$ & 26.11 & 15.21 & 53.50 & 16.72 & 53.55 & 13.98 & 64.99 & 10.95 & 70.94 \\
\hline & & & $(5.11)$ & $(3.90)$ & & $(4.09)$ & & $(3.74)$ & & $(3.31)$ & \\
\hline 9 & Untreated & -- & 24.70 & 32.71 & 0.00 & 36.00 & 0.00 & 39.94 & 0.00 & 37.69 & 0.00 \\
\hline & & & $(4.97)$ & $(5.72)$ & & $(6.00)$ & & $(6.32)$ & & $(6.14)$ & \\
\hline & $\mathrm{CD}(\mathrm{P}=0.05)$ & & NS & 0.33 & & 0.36 & & 0.33 & & 0.39 & \\
\hline & $\mathrm{SE}(\mathrm{m}) \pm$ & & 0.25 & 0.11 & & 0.12 & & 0.11 & & 0.13 & \\
\hline
\end{tabular}

DBS-Days before spraying, DAS-Days after spraying, NS-Non significant *figures in parentheses $\sqrt{x+0.5} \sqrt{x+0.5}$ transformed values 
Fig.1 Efficacy of bio- rational insecticides on the mean population of aphid (Aphis gossypi) in chilli crop after first spray

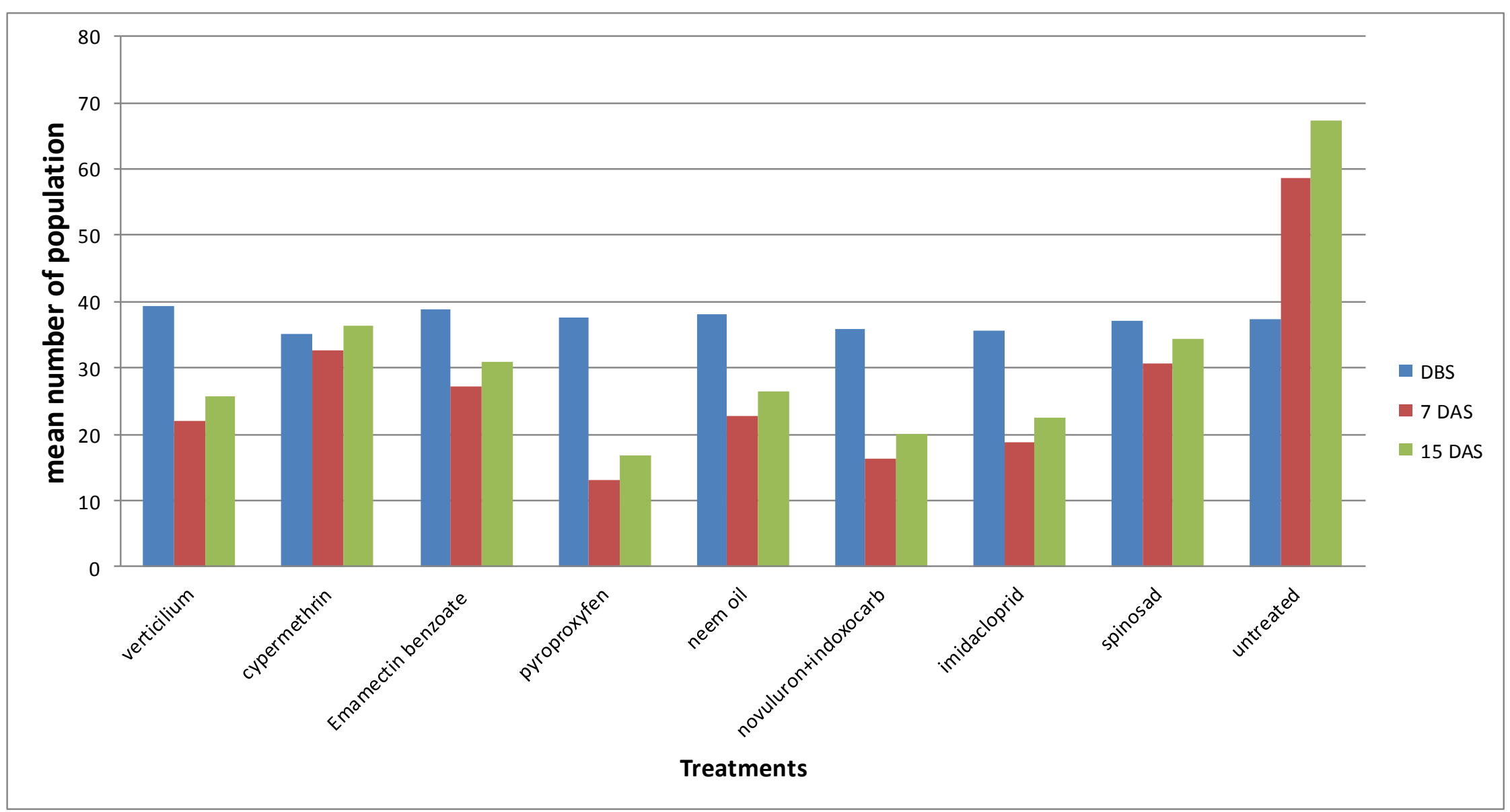


Fig.2 Efficacy of bio- rational insecticides on the mean population of aphid (Aphis gossypi) in chilli crop after second spray

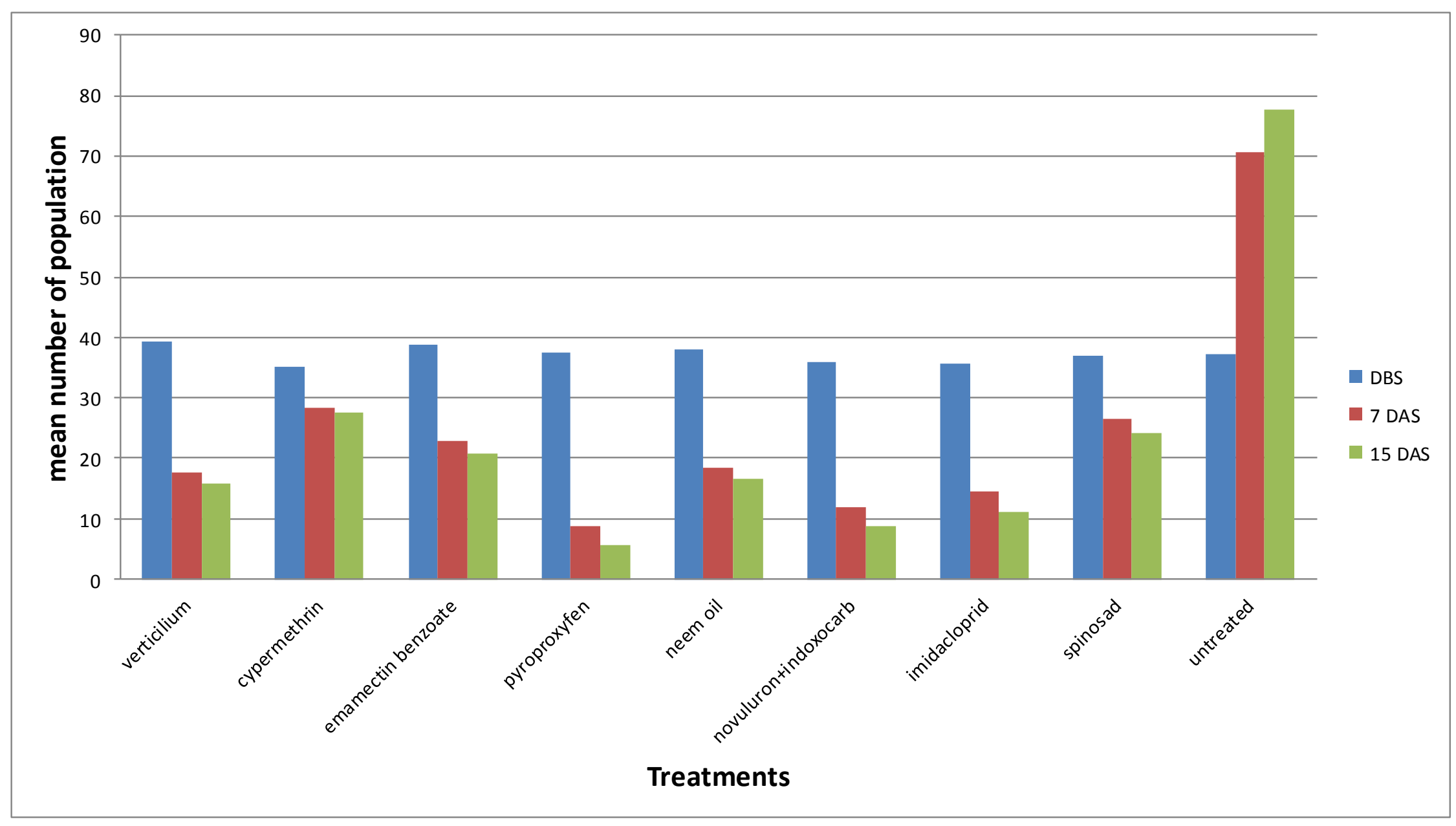


Fig.3 Efficacy of bio-rational insecticides on mean population of thirps (Scirtothirps dorsalis Hood) after first spray

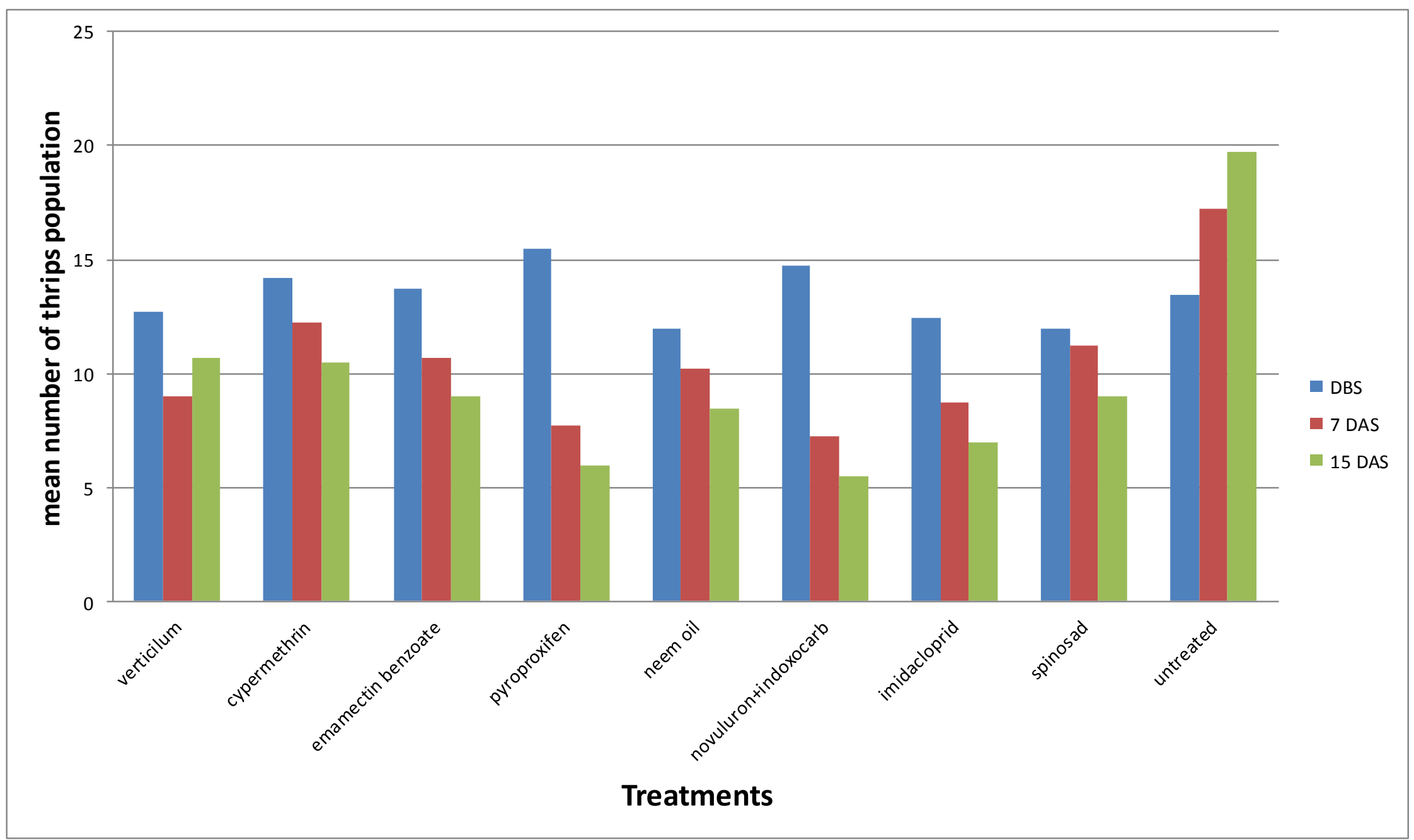


Fig.4 Efficacy of bio-rational insecticides on the mean population of thrips (Scirtothirps dorsalis Hood) after second spray

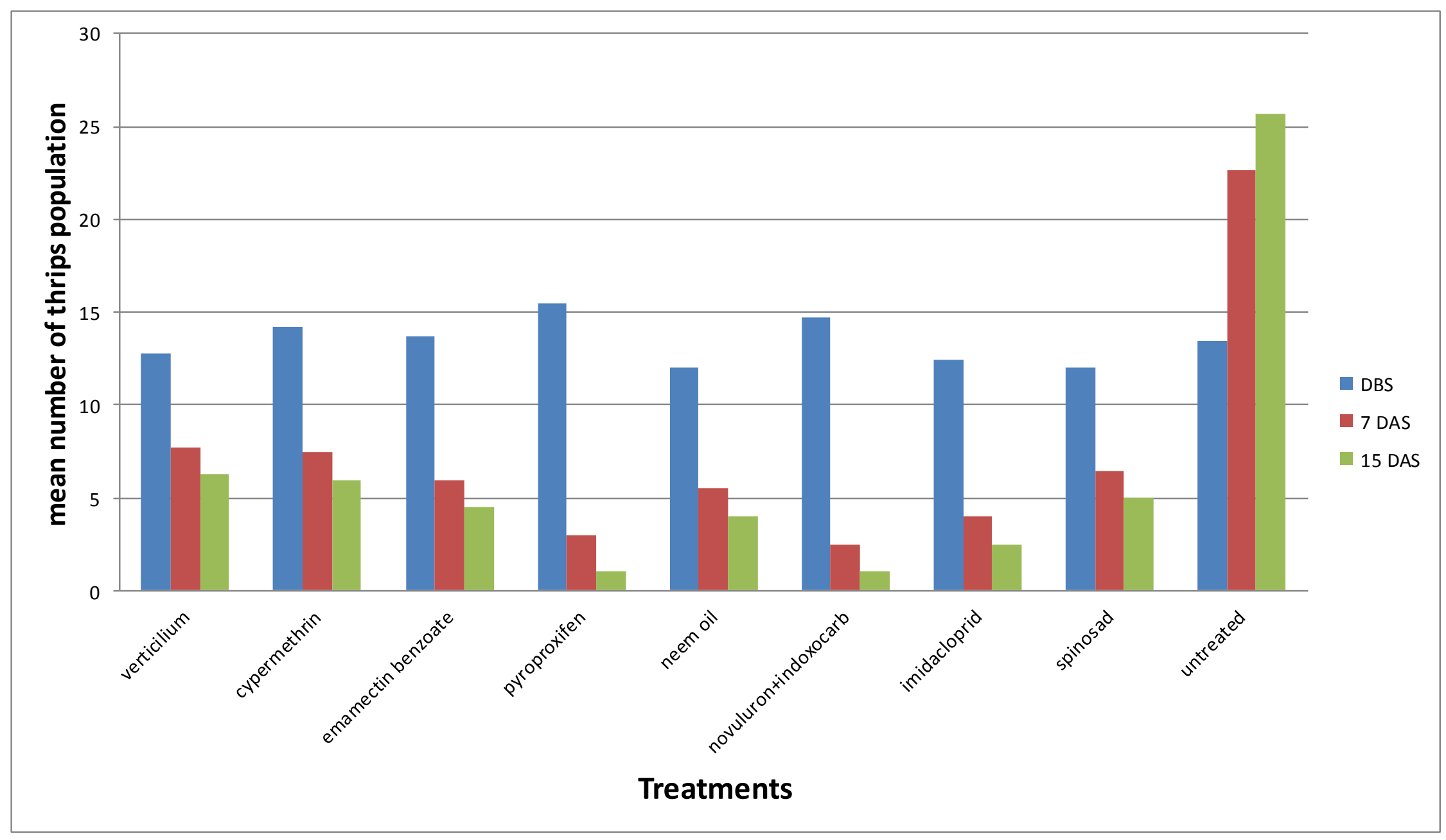


Fig. 5 Efficacy of bio-rational insecticides on the mean population of mites (Polyphagotarsonemus latus) after first spray

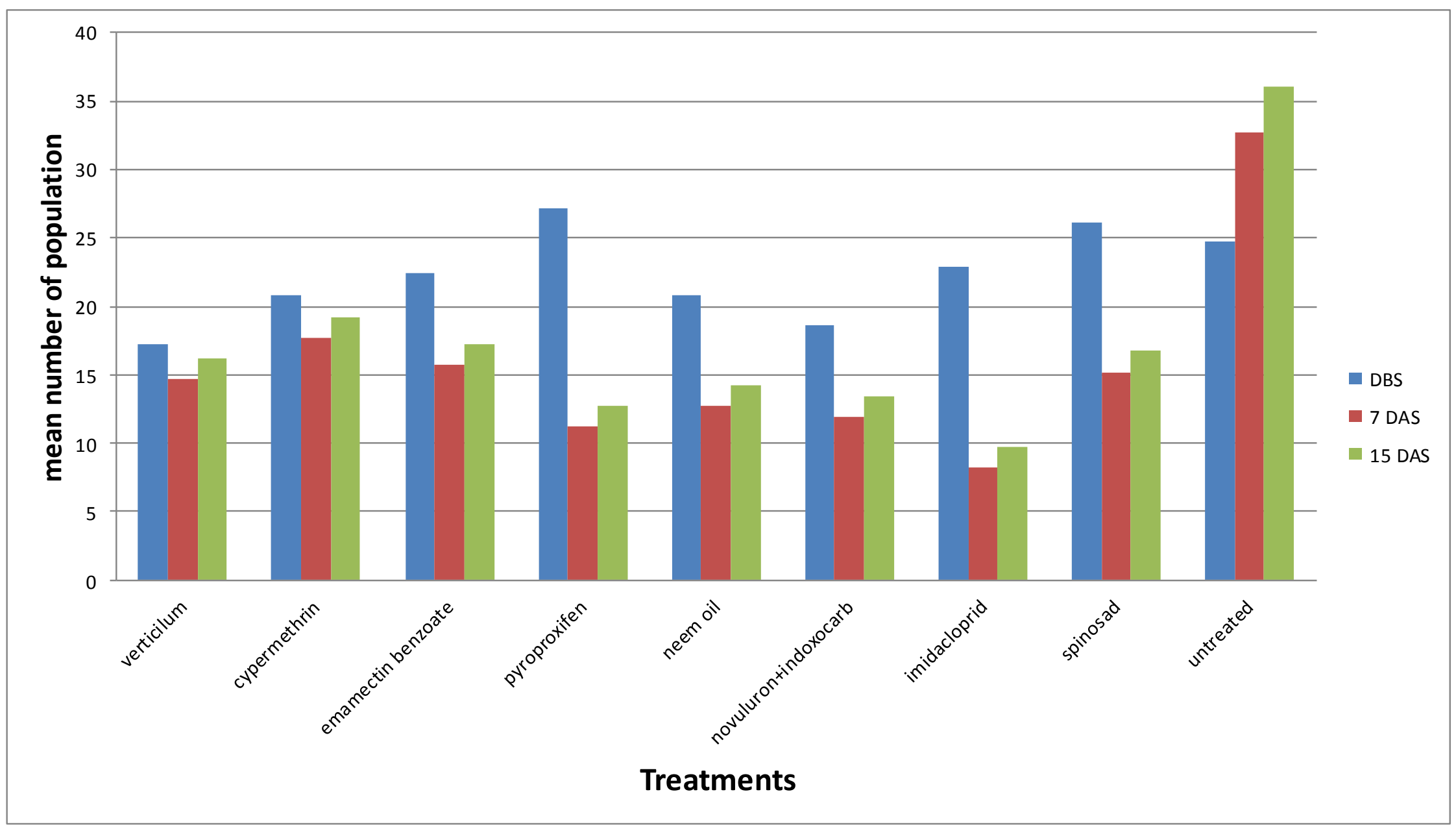


Fig. 6 Efficacy of bio-rational insecticides on the mean population of mite (Polyphagotarsonemus latus) after second spray

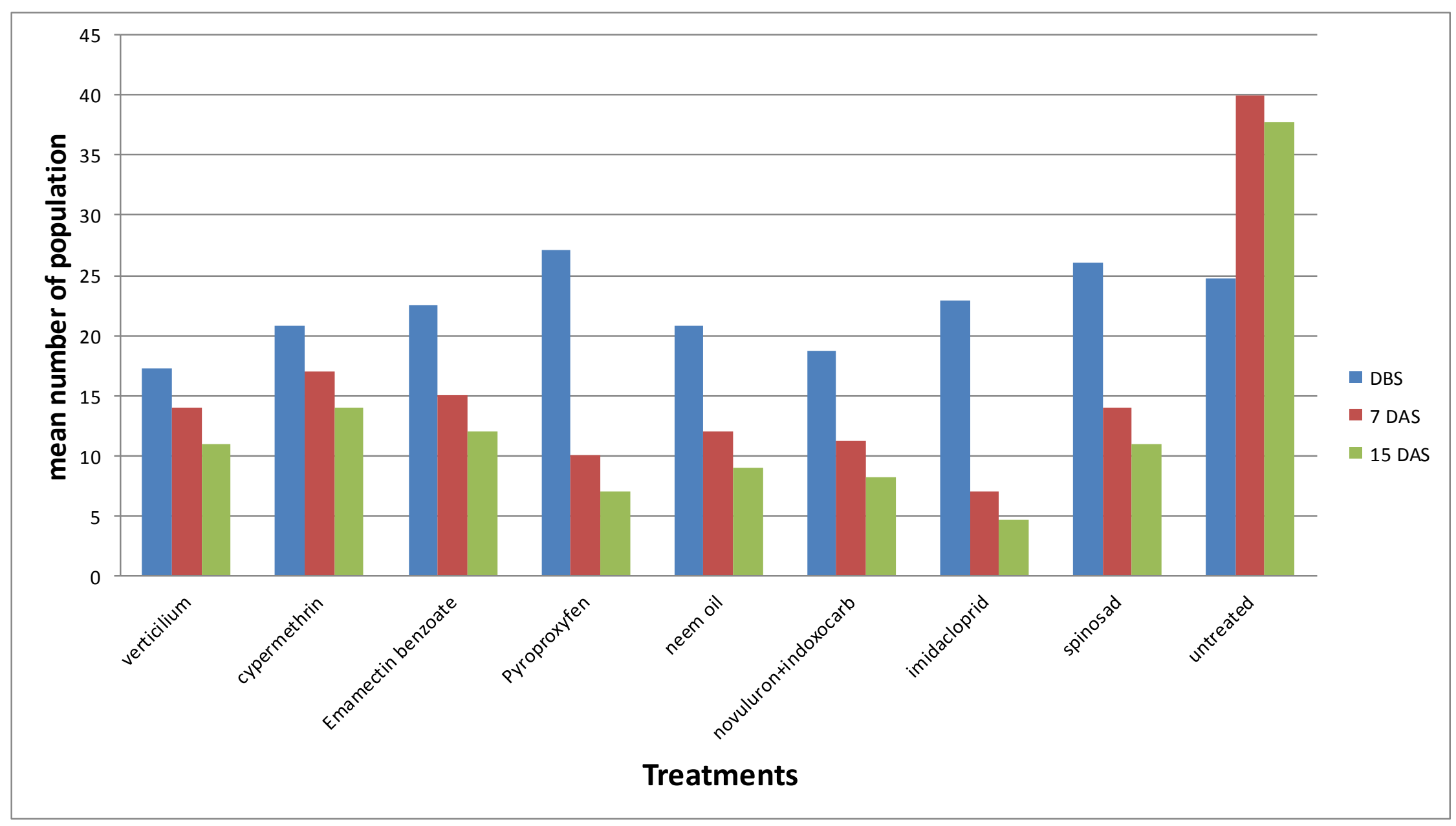


Efficacy of bio-rational insecticides on mites (P. latus)

A perusal of data from Table 2 and depicted in Fig. 1 indicate that there was no significant difference in pre-count of mite population in experimental plots showing homogenous pest distribution all over the plot. The mite population before spray ranged from 17.22 to 27.14 mites/ 3 leaves.

\section{Effect of First spray}

The data on the mean population of mites per plant is recorded after 7 and 15 of application. Among all the treatments imidacloprid @ $2.5 \mathrm{ml} / 1$ has the superior effect on controlling the mite population with mean population of $8.23 \mathrm{mites} / 3$ leaves and reduction percentage of 74.83 percent. Followed by pyroproxyfen @ $0.4 \mathrm{mg} / \mathrm{l}$ with mean mite population of $11.22 \mathrm{mites} / 3$ leaves and reduction percentage of $65.69 \%$.

\section{Effect of second spray}

Second spraying was done after 15 days of first spraying. The data is recorded 7 days after second spraying. The obtained data shows the following results. All the treatments are significantly superior over untreated control.

Imidacloprid @ $0.25 \mathrm{ml} / 1$ shows its superiority with mean population of 6.96 mites $/ 3$ leaves and has the reduction percentage of 82.57. After, this treatment pyroproxyfen@0.5mg/l, novuluron + indoxocarb @ 0.2ml $/ 1$, neem oil @ 0.5ml/1 obtained 75.01, 71.90, 70.03 percent of reduction in mite population over untreated control, respectively. The obtained results are at par with research work done by Latha and Hanumanythraya (2018), Manjunath et al., (2000) showed imdacloprid treatment showed significant reduction in mite population and higher yields in chilli.

\section{Effect of bio-rational insecticides on thrips (S. Dorsallis)}

\section{Effect of first spray}

Later treatments Novuluron + indoxocarb @ $0.2 \mathrm{ml} / 1$ and pyroproxyfen $@ 0.5 \mathrm{~g} / \mathrm{l}$ are also effective with reduction percentage of 58.01 and 55.61 over untreated control. imidacloprid@0.25ml/l, neem oil@5ml $/ 1$, verticilium@5g/l proved to be moderately effective with reduction percentage of 49.47 , $40.53,47.73$ over control plot respectively. Verticilium@5g/l, Cypermethrin@0.5ml/1 showed inferior performance but significantly better than untreated control.

\section{Effect of Second spray}

All the treatments are significantly superior over untreated plots. Novuluron + indoxocarb@0.2ml/1 had its superiority and has 89 percent of reduction in thrips population. pyroproxyfen@0.5mg/l and Imidacloprid@0.25ml/1 obtained 86.79 and 82.33 respectively over control. Neem oil@5m1/l, emamectin benzoate@0.4ml $/$, spinosad@0.5ml/1 was 75.84, 73.3, 71.52 percent of reduction. Verticilium@5g/l and Cypermethrin @0.5ml $/ 1$ had showed less effective. After 15 days of the spray of treatments, percentage of 96.10, Imidacloprid@0.25m1/l proved to be the next most effective, recorded 90.31 percent of reduction. seal et.al., (2006) reported imidicloprid is effective in thirps management. Maximum per cent reduction in aphids $(90.04 \%)$, thrips $(98.78 \%)$ by spraying of pyriproxifen $10 \% \mathrm{EW}$, Shivaray Nav et al., (2018).

\section{References}

Berke, T. and Sheih, S. C. 2000. Chilli peppers in Asia. Capsicum and Egg Plant Newslett., 19: 38-41. 
Gill, H. S., 1989. Improved technologies for chilli production. Indian Cocoa Arecanut and spices Journal, 12: 118119.

Gundannavar K P, Giraddi R S, Kulkarani K A, Awaknavar J S., 2007. Development of integrated pest management modules for chilli pests. Karnataka Journal of Agriculture Science, 20(4):757-760.

Hemant Swami, Lekha, Virender Singh, Deepak Jain and Kuldeep Kumar. 2018, Bio efficacy of pyriproxyfen $10 \%$ EC against whitefly, Bemisia tabaci and Aphids, Aphis gossipii infesting chilli crop, Journal of Entomology and Zoology Studies 2018; 6(4): 629-63.

Joia B S, Jaswinder K, Udean A S. Persistence of ethion residue on/in green chilli. Proceeding of National Symposium on Integrated Pest Management in Agriculture Crops, Bagalore, 2001, 174-175.

Kens, D. L., S. D. Stewart (2013), https://doi.org /10.1046/j.1570758.2000.00602.x

Kumar V, Swaminathan R, Singh H. 2015, Bio efficacy of newer insecticides against sucking insect pests of chilli. Annals of Plant Protection Science.; 23(1):69-73.

Manjunath M, Mallapur, C. P.; Hanchinal, S. G. ; Kulkarni, S. V., Karnataka Journal of Agricultural Sciences 2000 Vol.13 No.4 pp.993-995 ref.3.
Patel S. 2013, Studies on Insect Pest Complex of Brinjal, Solanum melongena (Linn.) and their control with insecticides and biopesticides. M.Sc. thesis, Jawaharlal Nehru Krishi Vishwa Vidyalaya, Jabalpur, India.

Patel, V. N. and Gupta, H. C. L. (1992). Investigation into the causes of leaf curl of chillies (Capsicum annum L.) in Rajasthan. Indian Journal of Applied Entomology, 6: 1-3.

Patil A S, Patil P D, Patil R S. 2002, Efficacy of different schedule doses of imidacloprid against sucking pests of chilli. Pestology.; 26(12):31.

Ratnakumari, P. V. L., Prabhu Prasadini, P., Venkat reddy, P., 2001, Active root distribution zone of Ball Paper (Capsicum annuun L.) under drip irrigation with and without mulches. Vegetable Science, 28 (1):82-83.

Seal D R, Ciomperlikb M, Richardsc M L, Klassena W. 2006; Comparative effectiveness of chemical insecticides against the chilli thrips, Scirtothrips dorsalis Hood (Thysanoptera: Thripidae), on pepper and their compatibility with natural enemies. Crop Protection. 25:949-955.

Shivaray Navi, Shashikumar C, Somu G, Meena N, Krishna Kishore R and Rajendra B, International Journal of Chemical Studies 2021; 9(1): 13131316.

\section{How to cite this article:}

Prathyusha, P., R. K. Dwivedi, Ajay deep singh and Gulab singh. 2021. Efficacy of BioRational Insecticides against Sucking Pest of Chilli. Int.J.Curr.Microbiol.App.Sci. 10(05): 546559. doi: https://doi.org/10.20546/ijcmas.2021.1005.062 